\title{
The Tamarkin equiconvergence theorem and a first-order trace formula for regular differential operators revisited
}

\author{
Alexander I. Nazarov, ${ }^{1}$ Dmitry M. Stolyarov, ${ }^{2,3}$ and Pavel B. Zatitskiy ${ }^{3}$ \\ Dedicated to the centenary of Boris Moiseevich Levitan
}

\begin{abstract}
We obtain a simple formula for the first-order trace of a regular differential operator on a segment perturbated by a multiplication operator. The main analytic ingredient of the proof is an improvement of the Tamarkin equiconvergence theorem.
\end{abstract}

Mathematics Subject Classification (2010). 34L05.

Keywords. Equiconvergence, regularized traces.

\section{Introduction}

1.1. Historical remarks. Consider a formal differential expression of the order $n \geqslant 2$,

$$
\ell:=(-i)^{n} D^{n}+\sum_{k=0}^{n-2} p_{k}(x) D^{k},
$$

acting on functions on some segment $[a, b]$ ( $D$ denotes differentiation with respect to $x$ ). We assume $p_{k}$ to be summable functions. Let $P_{j}$ and $Q_{j}, j \in\{0, \ldots, n-1\}$, be polynomials whose degrees do not exceed $n-1$. Then one can form the boundary conditions:

$$
P_{j}(D) y(a)+Q_{j}(D) y(b)=0, \quad j \in\{0, \ldots, n-1\},
$$

where $y$ is an arbitrary function.

\footnotetext{
${ }^{1}$ The first author is supported by the St. Petersburg State University grant 6.38 .64 .2012 and by the RFBR grant 13-01-00172.

${ }^{2}$ The second author is supported by RFBR grant 11-01-00526.

${ }^{3}$ The second and the third authors are supported by the Chebyshev Laboratory (SPbU), RF Government grant 11.G34.31.0026, and by the JSC "Gazprom Neft."
} 
Let $d_{j}, j \in\{0, \ldots, n-1\}$, be the maximum of degrees of $P_{j}$ and $Q_{j}$. Suppose $a_{j}$ and $b_{j}$ are the $d_{j}$-th coefficients of $P_{j}$ and $Q_{j}$ respectively. We assume that the system of boundary conditions (2) is normalized, i.e. $\sum_{j} d_{j}$ is minimal among all the systems of boundary conditions that can be obtained from (2) by linear bijective transformations. See [13], Chapter II, §4, for a detailed explanation and [21] for a more advanced treatment. We call system (2) almost separated if after some permutation of the boundary conditions we have

$$
\begin{aligned}
& \text { for } n=2 m: \quad b_{j}=0 \text { if } j<m ; \quad a_{j}=0 \text { if } j \geqslant m \text {; } \\
& \text { for } n=2 m+1: \quad b_{j}=0 \text { if } j<m ; \quad a_{j}=0 \text { if } j>m ; \quad a_{m} b_{m} \neq 0 .
\end{aligned}
$$

Differential expression (1) and boundary conditions (2) generate an operator $\mathbb{L}$ (see [13], Chapter I, for this standard procedure). We assume these boundary conditions to be regular in the sense of Birkhoff (see [13], Chapter II, §4). We underline that we do not require our operator to be self-adjoint; in particular, all the coefficients may be non-real.

We observe that the operator $\mathbb{L}$ has purely discrete spectrum (see [13], Chapter I) and denote it by $\left\{\lambda_{N}\right\}_{N=1}^{\infty}$. We always enumerate points of a spectrum in ascending order of their absolute values according to the multiplicity of eigenvalues, e.g., we assume that $\left|\lambda_{N}\right| \leqslant\left|\lambda_{N+1}\right|$.

Let $\mathbb{Q}$ be an operator of multiplication by a function $q \in L^{1}([a, b])$. Then, $\mathbb{L}+\mathbb{Q}$ also has purely discrete spectrum $\left\{\mu_{N}\right\}_{N=1}^{\infty}$.

In the previous paper [15], the authors obtained a formula for the first order trace

$$
\sum_{N=1}^{\infty}\left(\mu_{N}-\lambda_{N}\right)
$$

in terms of degrees of $P_{j}$ for the case of a self-adjoint semibounded operator with discrete spectrum on the halfline $\mathbb{R}_{+}$. We note that series (3) converges if and only if $\int q=0$, see Theorem 1 in [17], otherwise one has to regularize the trace to get something worth counting. Particular cases of this problem were considered earlier in papers [6], [17], [7], and in our preprint [14].

We conjectured that a similar formula should be valid for the case of an operator on an interval, at least if the boundary conditions are almost separated. This is really the case, though the details are dramatically different. In [15] we used the theorem on asymptotic behavior of the spectral functions of $\mathbb{L}$ and of the operator generated by the truncated expression and the same boundary conditions (2) obtained in [8] and [9]. Surprisingly, for the case of an interval the corresponding result was not known yet! So we had to prove this theorem, which refines the classical equiconvergence result of Tamarkin (see [24] or Theorem 1.5 in [12]; for the second-order operators see earlier papers [22], [23], [5], and [3]).

Theory of regularized traces originated in the fifties. We refer the reader to the survey [18] for the general historical scenery of the subject. We mention only several 
results that are closely related to our one. The first paper where such problems were considered was [2]. In this article the formula of regularized trace was calculated for the perturbation of a self-adjoint second order operator by a multiplication operator. Some particular cases of the fourth order operators were treated in [4], [10], and [1]. Operators of an arbitrary order without lower-order coefficients were considered in [20], where a formula for regularized trace was obtained for general Birkhoff regular boundary conditions. However, we should mention that the paper [20] deals with the case of a more regular function $q$ and does not provide short answers for the cases of almost separated and quasi-periodic boundary conditions. In [16], selfadjoint operators of an even order with lower order coefficients and special boundary conditions were considered. Namely, all derivatives of an even order were assumed to vanish on both ends of the interval. Formulas for $\delta(q)$ and for traces of higher order were given in terms of zeta function. It is worth to note also the paper [19] where the authors considered the second order operators with distribution potentials.

1.2. Setting of the problem and formulation of results. Let $\mathbb{L}_{0}$ be the operator generated by the differential expression $(-i)^{n} D^{n}$ and boundary conditions (2). Denote by $\left\{\lambda_{N}^{0}\right\}_{N=1}^{\infty}$ the eigenvalues of $\mathbb{L}_{0}$. Consider also the Green functions of operators $\mathbb{L}_{0}-\lambda$ and $\mathbb{L}-\lambda$, which we denote by $G_{0}(x, y, \lambda)$ and $G(x, y, \lambda)$, respectively. Then our main estimate reads as follows.

Theorem 1. For every sequence $R=R_{l} \rightarrow \infty$ separated from $\left|\lambda_{N}^{0}\right|^{\frac{1}{n}}$ the integral

$$
\int_{|\lambda|=R^{n}}\left|\left(G_{0}-G\right)(x, y, \lambda)\right||d \lambda|
$$

tends to zero uniformly with respect to $x, y \in[a, b]$.

This theorem is a generalization of the celebrated Tamarkin equiconvergence theorem mentioned above. Denote by $\theta_{R}(x, y)$ the integral

$$
\int_{|\lambda|=R^{n}}\left(G_{0}-G\right)(x, y, \lambda) d \lambda .
$$

Then the Tamarkin theorem states that the integral operator with the kernel $\theta_{R}$ acting from $L^{1}$ to $L^{\infty}$ tends to zero in the strong operator topology. Theorem 1 implies the same convergence in the norm operator topology. Though we had found this theorem during our study of regularized traces, it is interesting in itself.

Now we turn to the traces. Unfortunately, a beautiful formula similar to that of [15] does not hold for the general problem. So, we need to introduce some notation. 
Let $v_{1}=\left[\frac{n+1}{2}\right]$ and $v_{2}=\left[\frac{n}{2}\right]$. For $\kappa=1,2$ denote by $\widehat{W}^{[\kappa]}$ the matrix

$$
\widehat{W}^{[\kappa]}=\left(\begin{array}{cccccc}
a_{0} & \ldots & \rho^{\left(v_{\kappa}-1\right) d_{0}} a_{0} & \rho^{v_{\kappa} d_{0}} b_{0} & \ldots & \rho^{(n-1) d_{0}} b_{0} \\
\vdots & \vdots & \vdots & \vdots & & \vdots \\
a_{n-1} & \ldots & \rho^{\left(v_{\kappa}-1\right) d_{n-1}} a_{n-1} & \rho^{v_{\kappa} d_{n-1}} b_{n-1} & \ldots & \rho^{(n-1) d_{n-1}} b_{n-1}
\end{array}\right)
$$

(here and further $\rho=e^{\frac{2 \pi i}{n}}$ ). Note that these matrices are non-degenerate by the Birkhoff regularity condition.

Next, define matrices $\mathcal{A}$ and $\mathcal{B}$ with entries

$$
\mathcal{A}_{j k}=a_{j-1}\left(\rho^{k-1}\right)^{d_{j-1}}
$$

and

$$
\mathscr{B}_{j k}=b_{j-1}\left(\rho^{k-1}\right)^{d_{j-1}}
$$

for $j, k \in\{1, \ldots, n\}$. Finally, we introduce matrices $\mathcal{P}^{[\kappa]}$ and $\mathbb{Q}^{[\kappa]}=\left(\overline{\mathcal{P}}^{[\kappa]}\right)^{T}$, $\kappa=1,2$, by formulas

$$
\mathcal{P}_{\alpha \beta}^{[\kappa]}=\left\{\begin{array}{ll}
\frac{1}{\rho^{\beta-\alpha}-1}, & \alpha>v_{\kappa} \geqslant \beta ; \\
0, & \text { otherwise; }
\end{array} \quad \mathbb{Q}_{\alpha \beta}^{[\kappa]}= \begin{cases}\frac{1}{\rho^{\beta-\alpha}-1}, & \beta>v_{\kappa} \geqslant \alpha \\
0, & \text { otherwise. }\end{cases}\right.
$$

Note that if $n$ is even, then $v_{1}=v_{2}=\frac{n}{2}, \widehat{W}^{[1]}=\widehat{W}^{[2]}, \mathcal{P}^{[1]}=\mathcal{P}^{[2]}$, and $\mathcal{Q}^{[1]}=$ $Q^{[2]}$.

Now we can formulate the main result of our paper.

Theorem 2. Let $q \in L^{1}([a, b])$ be such that the functions

$$
\psi_{a}(x)=\frac{1}{x-a} \int_{a}^{x} q(t) d t-\frac{1}{b-a} \int_{a}^{b} q(t) d t
$$

and

$$
\psi_{b}(x)=\frac{1}{b-x} \int_{x}^{b} q(t) d t-\frac{1}{b-a} \int_{a}^{b} q(t) d t
$$

have bounded variation at the points $a$ and $b$, respectively. Then for the eigenvalues $\lambda_{N}$ and $\mu_{N}$ of the operators $\mathbb{L}$ and $\mathbb{L}+\mathbb{Q}$ defined above the following formula is 
true:

$$
\begin{aligned}
S(q) & \equiv \sum_{N=1}^{\infty}\left[\mu_{N}-\lambda_{N}-\frac{1}{b-a} \int_{a}^{b} q(t) d t\right] \\
& =\frac{\psi_{a}(a+)}{2 n} \cdot \sum_{\kappa=1}^{2} \operatorname{tr}\left(\mathcal{P}^{[\kappa]}\left(\hat{\mathfrak{W}}^{[\kappa]}\right)^{-1} \mathcal{A}\right)+\frac{\psi_{b}(b-)}{2 n} \cdot \sum_{\kappa=1}^{2} \operatorname{tr}\left(\mathcal{Q}^{[\kappa]}\left(\hat{\mathfrak{W}}^{[\kappa]}\right)^{-1} \mathfrak{B}\right) .
\end{aligned}
$$

Moreover, for $\kappa=1$ and $\kappa=2$ the following relation is true:

$$
\operatorname{tr}\left(\mathscr{P}^{[\kappa]}\left(\widehat{W}^{[\kappa]}\right)^{-1} \mathcal{A}\right)+\operatorname{tr}\left(\mathcal{Q}^{[\kappa]}\left(\widehat{W}^{[\kappa]}\right)^{-1} \mathscr{B}\right)=\sum_{j=0}^{n-1} d_{j}-\frac{n(n-1)}{2} .
$$

Remark 1. Formula (6) for $\mathbb{L}=\mathbb{Z}_{0}$ and for a smooth function $q$ was obtained in [20]. However, formula (7), as well as Theorem 3 below, is new even in this case.

For some classes of boundary conditions formula (6) can be considerably simplified.

Theorem 3. Let the assumptions of Theorem 2 be satisfied.

1. Suppose that boundary conditions (2) are almost separated. Then

1a) for $n=2 m$,

$$
\delta(q)=\frac{\psi_{a}(a+)}{2 m}\left(\sum_{j=0}^{m-1} d_{j}-\frac{m(2 m-1)}{2}\right)+\frac{\psi_{b}(b-)}{2 m}\left(\sum_{j=m}^{2 m-1} d_{j}-\frac{m(2 m-1)}{2}\right) ;
$$

1b) for $n=2 m+1$,

$$
\begin{aligned}
S(q)= & \frac{\psi_{a}(a+)}{2 m+1}\left(\sum_{j=0}^{m-1} d_{j}+\frac{d_{m}}{2}-\frac{m(2 m+1)}{2}\right) \\
& +\frac{\psi_{b}(b-)}{2 m+1}\left(\sum_{j=m+1}^{2 m} d_{j}+\frac{d_{m}}{2}-\frac{m(2 m+1)}{2}\right) .
\end{aligned}
$$

2. Suppose that the boundary conditions (2) are quasi-periodic, i.e. $d_{j}=j$ and $b_{j}=a_{j} \vartheta(\vartheta \neq 0)$ for $j \in\{0, \ldots, n-1\}$. Then

$$
\delta(q)=0 .
$$


The plan of our paper is as follows. In Section 2 we prove Theorem 1, almost by direct computation. Here we also establish auxiliary estimates which are used in the next section. In Subsection 3.1 we deduce formula (6) from Theorem 1. To do this, we improve the idea of [20]. Finally, in Subsection 3.2 we derive formulas (7)-(10) using technique and tricks similar to those we used in [15].

Acknowledgements. The authors are grateful to A. Minkin for his helpful advice and attracting our attention to the monograph [12]. We also thank A. Shkalikov for useful comments and D. Apushkinskaya for English language help.

\section{Proof of Theorem 1}

Throughout the paper we use the following notation. For $\lambda \in \mathbb{C}$ we define $z=\lambda^{\frac{1}{n}}$, $(\operatorname{Arg}(z) \in[0,2 \pi / n))$. For a function $\Phi$ defined on $\mathbb{C}$, we write $\widetilde{\Phi}(z)=\Phi(\lambda)$.

2.1. Formula for the Green function. We begin with finding the explicit formula for the Green function of $\mathbb{L}_{0}-\lambda$. We introduce a fundamental solution for the operator generated by $(-i)^{n} D^{n}-\lambda$ :

$$
\tilde{K}_{0}(x, y, z)= \begin{cases}0, & a \leqslant x<y \leqslant b ; \\ \frac{i}{n z^{n-1}} \sum_{k=0}^{n-1} \rho^{k} e^{i z \rho^{k}(x-y)}, & a \leqslant y \leqslant x \leqslant b .\end{cases}
$$

We search $\widetilde{G}_{0}$ as

$$
\widetilde{G}_{0}(x, y, z)=\tilde{K}_{0}(x, y, z)-\frac{i}{n z^{n-1}} \sum_{k=0}^{n-1} c_{k}(y, z) e^{i z \rho^{k}(x-y)} .
$$

We want to find functions $c_{k}$ such that the boundary relations (2) are fulfilled for $\widetilde{G}_{0}$ :

$$
\mathcal{W}(z) \cdot\left(\begin{array}{c}
c_{0}(y, z) \\
\vdots \\
e^{-i z \rho^{j-1} y} c_{j-1}(y, z) \\
\vdots \\
e^{-i z \rho^{n-1} y} c_{n-1}(y, z)
\end{array}\right)=\sum_{k=0}^{n-1} \rho^{k} e^{-i z y \rho^{k}} \cdot\left(\begin{array}{c}
e^{i z b \rho^{k}} Q_{0}\left(i z \rho^{k}\right) \\
\vdots \\
e^{i z b \rho^{k}} Q_{j}\left(i z \rho^{k}\right) \\
\vdots \\
e^{i z b \rho^{k}} Q_{n-1}\left(i z \rho^{k}\right)
\end{array}\right),
$$

where $\mathcal{W}(z)$ is a matrix containing the boundary values of the exponents:

$$
W_{j k}(z)=e^{i z \rho^{k-1} a} P_{j-1}\left(i z \rho^{k-1}\right)+e^{i z \rho^{k-1} b} Q_{j-1}\left(i z \rho^{k-1}\right), \quad j, k \in\{1, \ldots, n\} .
$$


We solve this linear equation using Cramer's rule:

$$
c_{\beta-1}(y, z)=\sum_{\alpha=1}^{n} \rho^{\alpha-1} e^{i z y\left(\rho^{\beta-1}-\rho^{\alpha-1}\right)} \cdot \frac{\Delta_{\alpha, \beta}(z)}{\Delta(z)} .
$$

Here $\Delta$ is the determinant of $\mathcal{W}, \Delta_{\alpha, \beta}$ is the determinant of a matrix that coincides with $\mathcal{W}$ except the column $\beta$ that is replaced by the $\alpha$-th column from the sum on the right-hand side of (11). Note that this changed column contains only the second summand of the $\alpha$-th column of $\mathcal{W}$.

Finally, the formula for the Green function is

$$
\widetilde{G}_{0}(x, y, z)=\widetilde{K}_{0}(x, y, z)-\frac{i}{n z^{n-1}} \sum_{\alpha, \beta=1}^{n} \rho^{\alpha-1} e^{i z\left(\rho^{\beta-1} x-\rho^{\alpha-1} y\right)} \cdot \frac{\Delta_{\alpha, \beta}(z)}{\Delta(z)} .
$$

\subsection{Asymptotics of the Green function}

Lemma 1. Set

and

$$
\Gamma_{1}=\left\{w=e^{i \varphi}: \varphi \in\left(0, \frac{\pi}{n}\right)\right\}
$$

$$
\Gamma_{2}=\left\{w=e^{i \varphi}: \varphi \in\left(\frac{\pi}{n}, \frac{2 \pi}{n}\right)\right\}
$$

Then for every sequence $R_{l} \rightarrow+\infty$ such that $R_{l}$ is separated from $\left|\lambda_{N}^{0}\right|^{\frac{1}{n}}$ and for all $j \in\{0, \ldots, n-1\}$ the function

$$
R_{l}^{n-1-j} \cdot\left|\left(\widetilde{G}_{0}\right)_{x}^{(j)}\left(x, y, R_{l} w\right)\right|
$$

is uniformly bounded on $[a, b]^{2} \times\left(\Gamma_{1} \cup \Gamma_{2}\right)$. Further, for every $x \in[a, b]$ one has

$$
R_{l}^{n-1} \cdot \tilde{G}_{0}\left(x, y, R_{l} w\right) \longrightarrow 0, \quad R_{l} \rightarrow+\infty
$$

for a.e. $y \in[a, b]$ and a.e. $w \in \Gamma_{1} \cup \Gamma_{2}$. Moreover, the convergence is uniform on $C \times J$ for arbitrary compact set $J \subset \Gamma_{1} \cup \Gamma_{2}$ and for arbitrary compact set $C \subset[a, b]^{2}$ separated from the corners and the diagonal $\{x=y\}$.

In what follows, when we write some limit over $R$ tending to $+\infty$ we mean the limit over this sequence $R_{l}$.

We turn to the proof of Lemma 1. The first part of this lemma (uniform estimates for $\widetilde{G}_{0}$ and its derivatives) can be easily extracted from [13], $\$ 4$. However, to prove convergence to zero, one has to do more work. We deal with formula (12) and evaluate each summand on its own way. Since different summands are estimated in a different way, we have to consider several cases. 
Note that for $x<y$

$$
R^{n-1} \cdot \widetilde{G}_{0}(x, y, R w)=-\frac{i}{n w^{n-1}} \sum_{\alpha, \beta=1}^{n} \rho^{\alpha-1} e^{i R w\left(\rho^{\beta-1} x-\rho^{\alpha-1} y\right)} \cdot \frac{\Delta_{\alpha, \beta}(R w)}{\Delta(R w)}
$$

while for $x \geqslant y$

$$
\begin{aligned}
R^{n-1} \cdot \widetilde{G}_{0}(x, y, R w)= & \frac{i}{n w^{n-1}} \sum_{\alpha=1}^{n} \rho^{\alpha-1} e^{i R w \rho^{\alpha-1}(x-y)}\left(1-\frac{\Delta_{\alpha, \alpha}(R w)}{\Delta(R w)}\right) \\
& -\frac{i}{n w^{n-1}} \sum_{\alpha \neq \beta} \rho^{\alpha-1} e^{i R w\left(\rho^{\beta-1} x-\rho^{\alpha-1} y\right)} \cdot \frac{\Delta_{\alpha, \beta}(R w)}{\Delta(R w)} .
\end{aligned}
$$

We begin with asymptotics of the elements of the matrix $\mathcal{W}$. If $\operatorname{Re}\left(i w \rho^{k-1}\right)>0$, then

$$
\begin{aligned}
\mathcal{W}_{j k}(R w)= & e^{i R w \rho^{k-1} b}\left(i R w \rho^{k-1}\right)^{d_{j-1}} \\
& \times\left(b_{j-1}+O\left(\frac{1}{R}\right)+e^{i R w \rho^{k-1}(a-b)}\left(a_{j-1}+O\left(\frac{1}{R}\right)\right)\right) \\
= & e^{i R w \rho^{k-1} b}\left(i R w \rho^{k-1}\right)^{d_{j-1}} \cdot\left(b_{j-1}+o(1)\right), \quad R \rightarrow+\infty .
\end{aligned}
$$

If $\operatorname{Re}\left(i w \rho^{k-1}\right)<0$, then

$$
\begin{aligned}
\mathcal{W}_{j k}(R w)= & e^{i R w \rho^{k-1} a}\left(i R w \rho^{k-1}\right)^{d_{j-1}} \\
& \quad \times\left(a_{j-1}+O\left(\frac{1}{R}\right)+e^{i R w \rho^{k-1}(b-a)}\left(b_{j-1}+O\left(\frac{1}{R}\right)\right)\right) \\
= & e^{i R w \rho^{k-1} a}\left(i R w \rho^{k-1}\right)^{d_{j-1}} \cdot\left(a_{j-1}+o(1)\right), \quad R \rightarrow+\infty .
\end{aligned}
$$

We note that the " $O$ " estimates are uniform on $\Gamma_{1} \cup \Gamma_{2}$ and the " $O$ " estimates are uniform on $J$.

Now we should differ the cases of odd and even $n$. Consider the function

$$
v(w)= \begin{cases}v_{1}=\left[\frac{n+1}{2}\right], & w \in \Gamma_{1} ; \\ v_{2}=\left[\frac{n}{2}\right], & w \in \Gamma_{2} .\end{cases}
$$

Note that if $n$ is even, then $v(w)=\frac{n}{2}$ for $w \in \Gamma_{1} \cup \Gamma_{2}$. If $n$ is odd, then $v(w)=\frac{n+1}{2}$ for $w \in \Gamma_{1}$ and $v(w)=\frac{n-1}{2}$ for $w \in \Gamma_{2}$. This number $v(w)$ is characterized by the following property: if $k \leqslant v(w)$, then $\operatorname{Re}\left(i w \rho^{k-1}\right)<0$, while if $v(w)<k \leqslant n$, then $\operatorname{Re}\left(i w \rho^{k-1}\right)>0$. Thus, for $w \in \Gamma_{1} \cup \Gamma_{2}$ the inequality $\operatorname{Re}\left(i w \rho^{k-1}\right)<0$ holds for $k \in\{1, \ldots, v(w)\}$. 
Next, we write the asymptotics of the determinant $\Delta$. We introduce the function

$$
f(R w)=\sum_{k=1}^{v(w)}\left|e^{i R w \rho^{k-1}(b-a)}\right|+\sum_{k=v(w)+1}^{n}\left|e^{-i R w \rho^{k-1}(b-a)}\right|, \quad w \in \Gamma_{1} \cup \Gamma_{2} .
$$

Clearly, $f(R w) \rightarrow 0$ uniformly on compact subsets of $\Gamma_{1} \cup \Gamma_{2}$ as $R \rightarrow+\infty$.

We factorize common factors from each column and row of $\Delta$ and get (see [13], §4)

$$
\Delta(R w)=e^{i a R w \sum_{k=1}^{\nu} \rho^{k-1}+i b R w} \sum_{k=v+1}^{n} \rho^{k-1} \cdot(i R w)^{\sum_{j=0}^{n-1} d_{j}} \cdot \Xi(R w),
$$

where

$$
\Xi(R w)=\widehat{\Delta}+O\left(\frac{1}{R}\right)+O(f(R w))=\widehat{\Delta}+o(1), \quad R \rightarrow+\infty,
$$

while $v=v_{\kappa}$ and $\widehat{\Delta}=\widehat{\Delta}^{[\kappa]} \equiv \operatorname{det} \widehat{w}^{[\kappa]}$ for $w \in \Gamma_{\kappa}$. Here the " $O$ " estimates are uniform for $w \in \Gamma_{1} \cup \Gamma_{2}$ and the " $o$ " is uniform for $w \in J$. Recall that the determinants $\widehat{\Delta}$ are non-zero by the Birkhoff regularity condition. Moreover, since $\left(\lambda_{N}^{0}\right)^{\frac{1}{n}}$ are zeros of $\Delta(z)$, the function $\Xi(R w)$ is separated from zero for $R=R_{l}$ and $w \in \Gamma_{1} \cup \Gamma_{2}$ by our choice of the sequence $R_{l}$.

Now we can write the asymptotics of terms in (13) and (14).

Case 1: $\alpha=\beta \leqslant v$. We have, as $R \rightarrow \infty$,

$$
\begin{aligned}
& \Delta_{\alpha, \alpha}(R w) \\
& =e^{i R w\left(b \rho^{\alpha-1}-a \rho^{\alpha-1}\right)} e^{i a R w \sum_{k=1}^{\nu} \rho^{k-1}+i b R w \sum_{k=\nu+1}^{n} \rho^{k-1}} \cdot(i R w)^{\sum_{j=0}^{n-1} d_{j}} \cdot\left(\widehat{\Delta}_{\alpha, \alpha}+o(1)\right) .
\end{aligned}
$$

Here $\widehat{\Delta}_{\alpha, \alpha}$ is the determinant of a matrix that differs from $\widehat{\mathcal{W}}$ only in the $\alpha$-th column. Namely, there are numbers $\rho^{(\alpha-1) d_{j}} b_{j}$ instead of $\rho^{(\alpha-1) d_{j}} a_{j}$. Thus, we obtain

$$
\frac{\Delta_{\alpha, \alpha}(R w)}{\Delta(R w)}=e^{i R w\left(b \rho^{\alpha-1}-a \rho^{\alpha-1}\right)}\left(\frac{\widehat{\Delta}_{\alpha, \alpha}}{\widehat{\Delta}}+o(1)\right), \quad R \rightarrow \infty .
$$

For $x<y$ this implies

$$
e^{i R w\left(\rho^{\alpha-1} x-\rho^{\alpha-1} y\right)} \frac{\Delta_{\alpha, \alpha}(R w)}{\Delta(R w)}=O\left(e^{i R w \rho^{\alpha-1}(b-a+x-y)}\right)=o(1), \quad R \rightarrow \infty,
$$

if $(x, y) \neq(a, b)$. For $x \geqslant y$ we obtain, as $R \rightarrow+\infty$,

$$
\begin{aligned}
e^{i R w \rho^{\alpha-1}(x-y)}\left(1-\frac{\Delta_{\alpha, \alpha}(R w)}{\Delta(R w)}\right) & =e^{i R w \rho^{\alpha-1}(x-y)}+O\left(e^{i R w \rho^{\alpha-1}(b-a+x-y)}\right) \\
& =o(1),
\end{aligned}
$$

if $x \neq y$. Here the " $o$ " estimates are uniform for $(x, y, w) \in C \times J$. 
Case 2: $\alpha=\beta>v$. We consider $\Delta-\Delta_{\alpha, \alpha}$ and use the linearity of the determinant with respect to the $\alpha$-th column to get $e^{i R w \rho^{\alpha-1} a} P_{j-1}\left(i R w \rho^{\alpha-1}\right)$ in the $\alpha$-th column. Using the same asymptotic formulas, we obtain

$$
e^{i R w \rho^{\alpha-1}(x-y)} \frac{\Delta(R w)-\Delta_{\alpha, \alpha}(R w)}{\Delta(R w)}=O\left(e^{i R w \rho^{\alpha-1}(a-b+x-y)}\right), \quad R \rightarrow \infty .
$$

For $x \geqslant y$ this implies

$$
e^{i R w \rho^{\alpha-1}(x-y)}\left(1-\frac{\Delta_{\alpha, \alpha}(R w)}{\Delta(R w)}\right)=O\left(e^{i R w \rho^{\alpha-1}(a-b+x-y)}\right)=o(1), \quad R \rightarrow+\infty,
$$

if $(x, y) \neq(b, a)$. For $x<y$ we obtain, as $R \rightarrow+\infty$,

$$
\begin{aligned}
e^{i R w\left(\rho^{\alpha-1} x-\rho^{\alpha-1} y\right) \frac{\Delta_{\alpha, \alpha}(R w)}{\Delta(R w)}} & =-e^{i R w \rho^{\alpha-1}(x-y)}+O\left(e^{i R w \rho^{\alpha-1}(b-a+x-y)}\right) \\
& =o(1) .
\end{aligned}
$$

Here the " $o$ " estimates are uniform for $(x, y, w) \in C \times J$.

Case 3: $\alpha \neq \beta$. In this case we either directly use the same asymptotic formulas (but with the " $O$ " estimates) or subtract the $\alpha$-th column from the $\beta$-th one in $\Delta_{\alpha, \beta}$ to make the exponent in the $\beta$-th column smaller (our choice of the procedure depends on the sign of $\left.\operatorname{Re}\left(i a w \rho^{\alpha-1}\right)\right)$.

Subcase 3.1: $\boldsymbol{\alpha}, \boldsymbol{\beta} \leqslant \boldsymbol{v}$. In this case $\operatorname{Re}\left(i a w \rho^{\alpha-1}\right)<0$, so we directly use the asymptotic formulas and get

$$
\begin{aligned}
& \frac{\Delta_{\alpha, \beta}(R w)}{\Delta(R w)} \\
& \quad=e^{i R w\left(b \rho^{\alpha-1}-a \rho^{\beta-1}\right)}\left(\frac{\widehat{\Delta}_{\alpha, \beta}+O\left(\frac{1}{R}\right)+O(f(R w))}{\Xi(R w)}\right) \\
& \quad=e^{i R w\left(b \rho^{\alpha-1}-a \rho^{\beta-1}\right)}\left(\frac{\widehat{\Delta}_{\alpha, \beta}}{\widehat{\Delta}}+O\left(\frac{1}{R}\right)+O(f(R w))\right), \quad R \rightarrow+\infty .
\end{aligned}
$$

Here $\widehat{\Delta}_{\alpha, \beta}$ is the determinant of a matrix that resembles $\widehat{w}$. The only difference is that numbers $\rho^{(\alpha-1) d_{j}} b_{j}$ replace $\rho^{(\beta-1) d_{j}} a_{j}$ in the $\beta$-th column. The last equation in (16) holds because the denominator $\Xi$ is separated from zero. The " $O$ " estimates are uniform for $w \in \Gamma_{1} \cup \Gamma_{2}$. 
Subcase 3.2: $\alpha \leqslant v<\beta$. In this case $\operatorname{Re}\left(\right.$ iaw $\left.\rho^{\alpha-1}\right)<0$ again, so we directly use the asymptotic formulas and get

$$
\frac{\Delta_{\alpha, \beta}(R w)}{\Delta(R w)}=e^{i R w\left(b \rho^{\alpha-1}-b \rho^{\beta-1}\right)}\left(\frac{\widehat{\Delta}_{\alpha, \beta}}{\widehat{\Delta}}+O\left(\frac{1}{R}\right)+O(f(R w))\right), \quad R \rightarrow+\infty .
$$

Here $\widehat{\Delta}_{\alpha, \beta}$ is the determinant of a matrix that resembles $\widehat{w}$, the only difference is that numbers $\rho^{(\alpha-1) d_{j}} b_{j}$ replace $\rho^{(\beta-1) d_{j}} b_{j}$ in the $\beta$-th column. The " $O$ " estimates are uniform for $w \in \Gamma_{1} \cup \Gamma_{2}$.

Subcase 3.3: $\alpha, \beta>v$. In this case $\operatorname{Re}\left(i a w \rho^{\alpha-1}\right)>0$, so we subtract the $\alpha$-th column from the $\beta$-th one in $\Delta_{\alpha, \beta}$. Arguing in the same way as before, one gets

$$
\frac{\Delta_{\alpha, \beta}(R w)}{\Delta(R w)}=e^{i R w\left(a \rho^{\alpha-1}-b \rho^{\beta-1}\right)}\left(-\frac{\widehat{\Delta}_{\alpha, \beta}}{\widehat{\Delta}}+O\left(\frac{1}{R}\right)+O(f(R w))\right), \quad R \rightarrow+\infty .
$$

Here $\widehat{\Delta}_{\alpha, \beta}$ is the determinant of a matrix that resembles $\widehat{w}$, the only difference is that numbers $\rho^{(\alpha-1) d_{j}} a_{j}$ replace $\rho^{(\beta-1) d_{j}} b_{j}$ in the $\beta$-th column. The " $O$ " estimates are uniform for $w \in \Gamma_{1} \cup \Gamma_{2}$.

Subcase 3.4: $\alpha>\boldsymbol{v} \geqslant \beta$. In this case $\operatorname{Re}\left(\right.$ iaw $\left.\rho^{\alpha-1}\right)>0$ again, so we subtract the $\alpha$-th column from the $\beta$-th one in $\Delta_{\alpha, \beta}$. Arguing in the same way as before, one gets

$$
\frac{\Delta_{\alpha, \beta}(R w)}{\Delta(R w)}=e^{i R w\left(a \rho^{\alpha-1}-a \rho^{\beta-1}\right)}\left(-\frac{\widehat{\Delta}_{\alpha, \beta}}{\widehat{\Delta}}+O\left(\frac{1}{R}\right)+O(f(R w))\right), \quad R \rightarrow+\infty .
$$

Here $\widehat{\Delta}_{\alpha, \beta}$ is the determinant of a matrix that resembles $\widehat{w}$, the only difference is that numbers $\rho^{(\alpha-1) d_{j}} a_{j}$ replace $\rho^{(\beta-1) d_{j}} a_{j}$ in the $\beta$-th column. The " $O$ " estimates are uniform for $w \in \Gamma_{1} \cup \Gamma_{2}$.

In all subcases we obtain

$$
e^{i R w\left(\rho^{\beta-1} x-\rho^{\alpha-1} y\right)} \frac{\Delta_{\alpha, \beta}(R w)}{\Delta(R w)}=o(1), \quad R \rightarrow \infty,
$$

if $(x, y) \notin\{(a, a),(a, b),(b, a),(b, b)\}$. Here the "o" estimates are uniform in $(x, y, w) \in C \times J$.

Summing up the estimates of cases 1-3, we complete the proof of Lemma 1.

Remark 2. We note that for odd $n$ the numbers $\widehat{\Delta}$ and $\widehat{\Delta}_{\alpha, \beta}$ defined in the proof of Lemma 1 depend on $w$ since the number $v$ depends on $w$. But these numbers are constants on $\Gamma_{1}$ and $\Gamma_{2}$. For even $n$ these numbers are constants on $\Gamma_{1} \cup \Gamma_{2}$. 
2.3. Truncation of the operator. In this subsection we prove Theorem 1 . We write down an identity

$$
\left(G-G_{0}\right)(x, y, \lambda)=-\int_{a}^{b} G_{0}(x, t, \lambda) \sum_{k=0}^{n-2} p_{k}(t) G_{t}^{(k)}(t, y, \lambda) d t,
$$

where $p_{k}$ are the lower order coefficients of $\mathbb{L}$. It is a reformulation of the Hilbert identity for resolvents,

$$
\frac{1}{\mathbb{L}-\lambda}-\frac{1}{\mathbb{L}_{0}-\lambda}=\frac{1}{\mathbb{L}-\lambda}\left(\mathbb{L}_{0}-\mathbb{L}\right) \frac{1}{\mathbb{L}_{0}-\lambda},
$$

in terms of the Green functions.

We differentiate equation (20) $j$ times with respect to $x$ :

$$
G_{x}^{(j)}(x, y, \lambda)=\left(G_{0}\right)_{x}^{(j)}(x, y, \lambda)-\int_{a}^{b}\left(G_{0}\right)_{x}^{(j)}(x, t, \lambda) \sum_{k=0}^{n-2} p_{k}(t) G_{t}^{(k)}(t, y, \lambda) d t
$$

Next, we multiply the expressions for $G_{x}^{(j)}$ by $p_{j}(x)$, sum up the results, and achieve

$$
\begin{aligned}
& \sum_{j=0}^{n-2} p_{j}(x) G_{x}^{(j)}(x, y, \lambda) \\
& =\sum_{j=0}^{n-2} p_{j}(x)\left(G_{0}^{(j)}\right)_{x}(x, y, \lambda) \\
& \quad-\sum_{j=0}^{n-2} p_{j}(x) \int_{a}^{b}\left(G_{0}\right)_{x}^{(j)}(x, t, \lambda) \sum_{k=0}^{n-2} p_{k}(t) G_{t}^{(k)}(t, y, \lambda) d t .
\end{aligned}
$$

Now let $|\lambda|^{\frac{1}{n}}=R=R_{l}$ be taken from Lemma 1 . Then the derivatives of $G_{0}$ can be estimated with the help of the first part of Lemma 1, and we obtain

$$
\left\|\sum_{j=0}^{n-2} p_{j}(\cdot) G^{(j)}(\cdot, y, \lambda)\right\|_{1} \leqslant \frac{C}{|\lambda|^{\frac{1}{n}}}+\frac{C}{|\lambda|^{\frac{1}{n}}} \cdot\left\|\sum_{j=0}^{n-2} p_{j}(\cdot) G^{(j)}(\cdot, y, \lambda)\right\|_{1} .
$$

This implies

$$
\left\|\sum_{j=0}^{n-2} p_{j}(\cdot) G^{(j)}(\cdot, y, \lambda)\right\|_{1} \leqslant \frac{C}{|\lambda|^{\frac{1}{n}}} .
$$

We substitute this inequality into (21) and get a pointwise estimate

$$
\left|G_{x}^{(j)}(x, y, \lambda)\right| \leqslant \frac{C}{|\lambda|^{\frac{n-1-j}{n}}}+\frac{C}{|\lambda|^{\frac{n-j}{n}}} \leqslant \frac{C}{|\lambda|^{\frac{n-1-j}{n}}} .
$$


Now we are ready to estimate the difference of the spectral functions of $\mathbb{L}$ and $\mathbb{L}_{0}$. Note that by formula (20)

$$
\begin{aligned}
& \int_{|\lambda|=R^{n}}\left|\left(G-G_{0}\right)(x, y, \lambda)\right||d \lambda| \\
& \quad \leqslant \int_{\Gamma_{1} \cup \Gamma_{2}} \int_{a}^{b} R^{n}\left|\widetilde{G}_{0}(x, t, R w)\right| \cdot\left|\sum_{j=0}^{n-2} p_{j}(t) \widetilde{G}_{t}^{(j)}(t, y, R w)\right| d t|d w| .
\end{aligned}
$$

By formula (22), the integrand has a majorant

$$
M(t, w)=\mathrm{const} \sum_{j=0}^{n-2}\left|p_{j}(t)\right| .
$$

We fix an $\varepsilon>0$ and choose $\delta>0$ such that the integral of $M$ over the set of measure not more than $\delta$ is less than $\varepsilon$.

Next, we choose a compact set $C \subset[a, b]^{2}$ separated from the diagonal and the corners, such that the measure of the set $C_{x}=\{t \in[a, b]:(x, t) \notin C\}$ is not more than $\frac{\delta n}{2 \pi}$ uniformly with respect to $x$. Also we choose a compact set $J \subset \Gamma_{1} \cup \Gamma_{2}$ such that the measure of $\Gamma_{1} \cup \Gamma_{2} \backslash J$ is not more than $\frac{\delta}{b-a}$.

The integral over the set $\left([a, b] \backslash C_{x}\right) \times J$ tends to zero as $R \rightarrow \infty$ uniformly in $(x, y) \in[a, b]^{2}$, since by Lemma 1 and formula (22) the integrand tends to zero uniformly on this set. The integral over the remaining set does not exceed $2 \varepsilon$. Thus, for $R$ large enough, the whole integral is not bigger than $3 \varepsilon$ for all $(x, y) \in[a, b]^{2}$, and the theorem follows.

\section{Proof of Theorems 2 and 3}

3.1. Reduction to linear algebra. First of all, we can assume

$$
\int_{a}^{b} q(x) d x=0
$$

because adding a constant to $q$ only shifts the spectrum $\mu_{N}$, but does not change $\delta(q)$ and the right hand side of equations (6), (8), and (9). We begin with formula

$$
\sum_{\left|\lambda_{N}\right|<R^{n}} \lambda_{N}=-\frac{1}{2 \pi i} \int_{|\lambda|=R^{n}} \lambda \mathbf{S p} \frac{1}{\mathbb{L}-\lambda} d \lambda,
$$

where the trace on the right-hand side is an integral operator trace

$$
\mathbf{S p} \frac{1}{\mathbb{L}-\lambda}=\int_{a}^{b} G(x, x, \lambda) d x .
$$


Indeed, by the Lidskii theorem [11],

$$
\sum_{N} \frac{1}{\lambda_{N}-\lambda}=\mathbf{S p} \frac{1}{\mathbb{L}-\lambda}
$$

for all $\lambda$ which are not in the spectrum of $\mathbb{L}$ (we use the fact that the resolvent $\frac{1}{\mathbb{L}-\lambda}$ belongs to the trace class, because $\left|\lambda_{N}\right|$ grow as $N^{n}$ ). We multiply this equation by $\lambda$, integrate over the circle $|\lambda|=R^{n}$, use the residue theorem and arrive at (23).

Now we can express $S(q)$ using the Hilbert identity for resolvents:

$$
\begin{aligned}
S(q)= & \frac{1}{2 \pi i} \lim _{R \rightarrow \infty} \int_{|\lambda|=R^{n}} \lambda \mathbf{S p}\left(\frac{1}{\mathbb{L}-\lambda}-\frac{1}{\mathbb{L}+\mathbb{Q}-\lambda}\right) d \lambda \\
= & \frac{1}{2 \pi i} \lim _{R \rightarrow \infty} \int_{|\lambda|=R^{n}} \lambda \mathbf{S p}\left(\frac{1}{\mathbb{L}-\lambda} \mathbb{Q} \frac{1}{\mathbb{L}+\mathbb{Q}-\lambda}\right) d \lambda \\
= & -\frac{1}{2 \pi i} \lim _{R \rightarrow \infty} \frac{1}{2} \int_{|\lambda|=R^{n}} \lambda \mathbf{S p}\left(\left(\frac{1}{\mathbb{L}-\lambda}-\frac{1}{\mathbb{L}+\mathbb{Q}-\lambda}\right)\right. \\
& \left.+\frac{1}{2 \pi i} \lim _{R \rightarrow \infty} \frac{1}{2} \int_{|\lambda|=R^{n}} \lambda \mathbf{S}\left(\frac{1}{\mathbb{L}-\lambda}-\frac{1}{\mathbb{L}+\mathbb{Q}-\lambda}\right)\right) d \lambda \\
& \left.\quad+\frac{1}{\mathbb{Q}+\mathbb{Q}-\lambda} \frac{1}{\mathbb{L}-\lambda} \frac{1}{\mathbb{Q}+\mathbb{Q}-\lambda}\right) d \lambda .
\end{aligned}
$$

Obviously, we can take the limit over a sequence of $R$ separated from $\left|\lambda_{N}^{0}\right|^{\frac{1}{n}}$.

We claim that the first integral in the right-hand side of (24) disappears at infinity. Indeed, it can be estimated as follows:

$$
\begin{aligned}
& \int_{|\lambda|=} \lambda \mathbf{S p}\left(\left(\frac{1}{\mathbb{L}-\lambda}-\frac{1}{\mathbb{L}+\mathbb{Q}-\lambda}\right) \mathbb{Q}\left(\frac{1}{\mathbb{L}-\lambda}-\frac{1}{\mathbb{L}+\mathbb{Q}-\lambda}\right)\right) d \lambda \\
& \quad=\int_{|\lambda|=R^{n}} \lambda \mathbf{S p}\left(\left(\frac{1}{\mathbb{L}-\lambda} \mathbb{Q} \frac{1}{\mathbb{L}+\mathbb{Q}-\lambda}\right) \mathbb{Q}\left(\frac{1}{\mathbb{L}-\lambda} \mathbb{Q} \frac{1}{\mathbb{L}+\mathbb{Q}-\lambda}\right)\right) d \lambda \\
& =O\left(R^{2-\frac{4(n-1)}{n}}\right)
\end{aligned}
$$

by inequality (22). If $n>2$, then this value tends to zero. In the remaining case we replace the first $\frac{1}{\mathbb{L}-\lambda}$ in (25) by $\frac{1}{\mathbb{L}_{0}-\lambda}$. The difference tends to zero by Theorem 1 while the changed integral can be estimated with the help of the first part of Lemma 1 and the Lebesgue Dominated Convergence theorem in the same way as we did at the end of the proof of Theorem 1. Thus, the claim follows. 
The second integral can be transformed as follows:

$$
\begin{aligned}
& \int_{|\lambda|=R^{n}} \lambda \mathbf{S p}\left(\frac{1}{\mathbb{L}-\lambda} \mathbb{Q} \frac{1}{\mathbb{L}-\lambda}+\frac{1}{\mathbb{L}+\mathbb{Q}-\lambda} \mathbb{Q} \frac{1}{\mathbb{L}+\mathbb{Q}-\lambda}\right) d \lambda \\
& \quad=\int_{|\lambda|=R^{n}} \mathbf{S p}\left(\left(\frac{\lambda}{(\mathbb{L}-\lambda)^{2}}+\frac{\lambda}{(\mathbb{L}+\mathbb{Q}-\lambda)^{2}}\right) \mathbb{Q}\right) d \lambda \\
& =-\int_{|\lambda|=R^{n}} \mathbf{S p}\left(\left(\frac{1}{\mathbb{L}-\lambda}+\frac{1}{\mathbb{L}+\mathbb{Q}-\lambda}\right) \mathbb{Q}\right) d \lambda \\
& =-2 \int_{|\lambda|=R^{n}} \mathbf{S p}\left(\frac{1}{\mathbb{L}_{0}-\lambda} \mathbb{Q}\right) d \lambda+o(1), \quad R \rightarrow \infty .
\end{aligned}
$$

The first equality in (26) is the identity $\mathbf{S p}(A B C)=\mathbf{S p}(B C A)$, the second one is integration by parts, and the third one follows from Theorem 1 . Thus, we arrive at

$$
\begin{aligned}
S(q) & =-\frac{1}{2 \pi i} \lim _{R \rightarrow \infty} \int_{|\lambda|=R^{n}} \int_{a}^{b} q(x) G_{0}(x, x, \lambda) d x d \lambda \\
& =-\frac{1}{2 \pi i} \lim _{R \rightarrow \infty} \int_{R\left(\Gamma_{1} \cup \Gamma_{2}\right)} \int_{a}^{b} q(x) \widetilde{G}_{0}(x, x, z) n z^{n-1} d x d z \\
& =\frac{1}{2 \pi} \sum_{\alpha, \beta=1}^{n} I_{\alpha, \beta},
\end{aligned}
$$

where

$$
I_{\alpha, \beta}=\lim _{R \rightarrow \infty} \int_{R\left(\Gamma_{1} \cup \Gamma_{2}\right)} \int_{a}^{b} q(x) \rho^{\alpha-1} e^{i z x\left(\rho^{\beta-1}-\rho^{\alpha-1}\right)} \cdot \frac{\Delta_{\alpha, \beta}(z)}{\Delta(z)} d x d z .
$$

The last equality in (27) holds because of relation $\widetilde{K}_{0}(x, x, z)=0$.

If $\alpha=\beta$, the integral (28) equals zero by the assumption

$$
\int_{a}^{b} q(x) d x=0
$$

So we turn to the case $\alpha \neq \beta$. We use the asymptotic formulas for the quotients $\frac{\Delta_{\alpha, \beta}}{\Delta}$ obtained in the proof of Lemma 1. 
Denote by $I_{\alpha, \beta}^{[\kappa]}, \kappa=1,2$, the same limit as $I_{\alpha, \beta}$ but with the outer integral taken over $R \Gamma_{\kappa}$ instead of $R\left(\Gamma_{1} \cup \Gamma_{2}\right)$. Then $I_{\alpha, \beta}=I_{\alpha, \beta}^{[1]}+I_{\alpha, \beta}^{[2]}$. There are four subcases.

Subcase 1: $\alpha, \beta \leqslant v_{\kappa}$. We use (16) to write

$$
\begin{aligned}
& I_{\alpha, \beta}^{[\kappa]}= \frac{\widehat{\Delta}_{\alpha, \beta}^{[\kappa]}}{\widehat{\Delta}^{[\kappa]}} \cdot \rho^{\alpha-1} \lim _{R \rightarrow \infty} \int_{\Gamma_{\kappa}} \int_{a}^{b} R q(x) e^{i R w\left(\rho^{\beta-1}(x-a)+(b-x) \rho^{\alpha-1}\right)} d x d w \\
&+\rho^{\alpha-1} \lim _{R \rightarrow \infty} \int_{\Gamma_{\kappa}}(O(1)+O(R f(R w))) \\
& \quad \times \int_{a}^{b} q(x) e^{i R w\left(\rho^{\beta-1}(x-a)+(b-x) \rho^{\alpha-1}\right)} d x d w .
\end{aligned}
$$

The last term in (29) can be estimated as follows:

$$
\begin{aligned}
& \left|\int_{\Gamma_{\kappa}}(O(1)+O(f(R w))) \int_{a}^{b} q(x) e^{i R w\left(\rho^{\beta-1}(x-a)+(b-x) \rho^{\alpha-1}\right)} d x d w\right| \\
& \quad \leqslant \sup _{w \in \Gamma_{\kappa}}\left|\int_{a}^{b} q(x) e^{i R w\left(\rho^{\beta-1}(x-a)+(b-x) \rho^{\alpha-1}\right)} d x\right| \cdot \int_{\Gamma_{\kappa}}(O(1)+O(f(R w)))|d w| .
\end{aligned}
$$

The first factor tends to zero by Proposition 1 as $R \rightarrow \infty$, while the second one is bounded by Proposition 2 (see Appendix). Therefore, we obtain

$$
I_{\alpha, \beta}^{[\kappa]}=\frac{\widehat{\Delta}_{\alpha, \beta}^{[\kappa]}}{\widehat{\Delta}^{[\kappa]}} \cdot \rho^{\alpha-1} \lim _{R \rightarrow \infty} \int_{\Gamma_{\kappa}} \int_{a}^{b} R q(x) e^{i R w\left(\rho^{\beta-1}(x-a)+\rho^{\alpha-1}(b-x)\right)} d x d w .
$$

The same calculations for three other subcases give the following formulas.

Subcase 2: $\alpha \leqslant v_{\kappa}<\beta$. We have

$$
I_{\alpha, \beta}^{[\kappa]}=\frac{\widehat{\Delta}_{\alpha, \beta}^{[\kappa]}}{\widehat{\Delta}^{[\kappa]}} \cdot \rho^{\alpha-1} \lim _{R \rightarrow \infty} \int_{\Gamma_{\kappa}} \int_{a}^{b} R q(x) e^{i R w\left(\rho^{\alpha-1}-\rho^{\beta-1}\right)(b-x)} d x d w .
$$

Subcase 3: $\alpha, \beta>v_{\kappa}$. We have

$$
I_{\alpha, \beta}^{[\kappa]}=-\frac{\widehat{\Delta}_{\alpha, \beta}^{[\kappa]}}{\widehat{\Delta}^{[\kappa]}} \cdot \rho^{\alpha-1} \lim _{R \rightarrow \infty} \int_{\Gamma_{\kappa}} \int_{a}^{b} R q(x) e^{i R w\left(\rho^{\beta-1}(x-b)+\rho^{\alpha-1}(a-x)\right)} d x d w .
$$


Subcase 4: $\alpha>v_{\kappa} \geqslant \beta$. We have

$$
I_{\alpha, \beta}^{[\kappa]}=-\frac{\widehat{\Delta}_{\alpha, \beta}^{[\kappa]}}{\widehat{\Delta}^{[\kappa]}} \cdot \rho^{\alpha-1} \lim _{R \rightarrow \infty} \int_{\Gamma_{\kappa}} \int_{a}^{b} R q(x) e^{i R w\left(\rho^{\beta-1}-\rho^{\alpha-1}\right)(x-a)} d w d x .
$$

In Subcase 1 we integrate with respect to $w$ and obtain

$$
\begin{array}{r}
I_{\alpha, \beta}^{[\kappa]}=\frac{\widehat{\Delta}_{\alpha, \beta}^{[\kappa]}}{\widehat{\Delta}^{[\kappa]}} \cdot \rho^{\alpha-1} \lim _{R \rightarrow \infty} \int_{a}^{b} q(x)\left(\frac{e^{i R\left(\rho^{\beta-1}(x-a)+\rho^{\alpha-1}(b-x)\right)(\sqrt{\rho})^{\kappa}}}{i\left(\rho^{\beta-1}(x-a)+\rho^{\alpha-1}(b-x)\right)}\right. \\
\left.-\frac{e^{i R\left(\rho^{\beta-1}(x-a)+\rho^{\alpha-1}(b-x)\right)(\sqrt{\rho})^{\kappa-1}}}{i\left(\rho^{\beta-1}(x-a)+\rho^{\alpha-1}(b-x)\right)}\right) d x,
\end{array}
$$

where $\sqrt{\rho}=e^{\frac{i \pi}{n}}$. Here the denominator is uniformly separated from zero, and the numerator is uniformly bounded. Thus, the integrand has a summable majorant $C|q(x)|$. Moreover, since $\alpha \neq \beta$ and $\alpha, \beta \leqslant v_{\kappa}$, the numerator tends to zero for a.e. $x \in[a, b]$. By the Lebesgue Dominated Convergence theorem, $I_{\alpha, \beta}^{[\kappa]}=0$. The same arguments show that $I_{\alpha, \beta}^{[\kappa]}=0$ in Subcase 3 .

In Subcases 2 and 4 after integration with respect to $w$ the denominators are not separated from zero. So, we should use the regularity of $q$ at the endpoints. Namely, under assumptions of Theorem 2 the functions $\psi_{a}$ and $\psi_{b}$ belong to $W_{1}^{1}([a, b])$, and

$$
q(x)=\psi_{a}(x)+(x-a) \psi_{a}^{\prime}(x)=\psi_{b}(x)+(x-b) \psi_{b}^{\prime}(x) .
$$

Let us consider Subcase 4. Using the first equality in (34) we obtain

$$
\begin{aligned}
& I_{\alpha, \beta}^{[\kappa]}=- \frac{\widehat{\Delta}_{\alpha, \beta}^{[\kappa]}}{\widehat{\Delta}^{[\kappa]}} \cdot \rho^{\alpha-1} \lim _{R \rightarrow \infty} \int_{\Gamma_{\kappa}} \int_{a}^{b} R \psi_{a}(x) e^{i R w\left(\rho^{\beta-1}-\rho^{\alpha-1}\right)(x-a)} d x d w \\
&-\frac{\widehat{\Delta}_{\alpha, \beta}^{[\kappa]}}{\widehat{\Delta}^{[\kappa]}} \cdot \rho^{\alpha-1} \lim _{R \rightarrow \infty} \int_{a}^{b} \psi_{a}^{\prime}(x)\left(\frac{e^{i R\left(\rho^{\beta-1}-\rho^{\alpha-1}\right)(x-a)(\sqrt{\rho})^{\kappa}}}{i\left(\rho^{\beta-1}-\rho^{\alpha-1}\right)}\right. \\
&\left.-\frac{e^{i R\left(\rho^{\beta-1}-\rho^{\alpha-1}\right)(x-a)(\sqrt{\rho})^{\kappa-1}}}{i\left(\rho^{\beta-1}-\rho^{\alpha-1}\right)}\right) d x .
\end{aligned}
$$

Since $\alpha>v_{\kappa} \geqslant \beta$, the last limit equals zero by Proposition 1 . So, integrating by parts, we have

$$
\begin{aligned}
& I_{\alpha, \beta}^{[\kappa]}=-\frac{\hat{\Delta}_{\alpha, \beta}^{[\kappa]}}{\widehat{\Delta}^{[\kappa]}} \cdot \rho^{\alpha-1} \lim _{R \rightarrow \infty} \int_{\Gamma_{\kappa}}\left[\left.\psi_{a}(x) \frac{e^{i R w\left(\rho^{\beta-1}-\rho^{\alpha-1}\right)(x-a)}}{i w\left(\rho^{\beta-1}-\rho^{\alpha-1}\right)}\right|_{a} ^{b}\right. \\
& \left.-\int_{a}^{b} \psi_{a}^{\prime}(x) \frac{e^{i R w\left(\rho^{\beta-1}-\rho^{\alpha-1}\right)(x-a)}}{i w\left(\rho^{\beta-1}-\rho^{\alpha-1}\right)} d x\right] d w .
\end{aligned}
$$


The last term here also tends to zero by Proposition 1. Moreover, the term with substitution $x=b$ tends to zero by the Lebesgue Dominated Convergence theorem, and we arrive at

$$
I_{\alpha, \beta}^{[\kappa]}=\frac{\widehat{\Delta}_{\alpha, \beta}^{[\kappa]}}{\widehat{\Delta}^{[\kappa]}} \cdot \frac{\rho^{\alpha-1}}{i\left(\rho^{\beta-1}-\rho^{\alpha-1}\right)} \psi_{a}(a+) \cdot \int_{\Gamma_{\kappa}} \frac{d w}{w}=\frac{\pi}{n} \frac{\widehat{\Delta}_{\alpha, \beta}^{[\kappa]}}{\widehat{\Delta}^{[\kappa]}} \frac{\rho^{\alpha-1}}{\rho^{\beta-1}-\rho^{\alpha-1}} \psi_{a}(a+) .
$$

By Cramer's rule, for all $\alpha>v_{\kappa} \geqslant \beta$ we have

$$
\frac{\widehat{\Delta}_{\alpha, \beta}^{[\kappa]}}{\widehat{\Delta}^{[\kappa]}}=\left(\left(\widehat{W}^{[\kappa]}\right)^{-1} \mathcal{A}\right)_{\beta \alpha},
$$

and thus

$$
I_{\alpha, \beta}^{[\kappa]}=\frac{\pi}{n} \psi_{a}(a+) \cdot\left(\left(\hat{W}^{[\kappa]}\right)^{-1} \mathcal{A}\right)_{\beta \alpha} \mathcal{P}_{\alpha \beta}^{[\kappa]}, \quad \beta \leqslant v_{\kappa}<\alpha,
$$

where the matrix $\mathscr{P}^{[\kappa]}$ was introduced in (5).

Since $\mathcal{P}_{\alpha \beta}^{[\kappa]}=0$ for other pairs $(\alpha, \beta)$, we obtain

$$
\sum_{\alpha>v_{\kappa} \geqslant \beta} I_{\alpha, \beta}^{[\kappa]}=\frac{\pi}{n} \psi_{a}(a+) \cdot \operatorname{tr}\left(\mathcal{P}^{[\kappa]}\left(\hat{W}^{[\kappa]}\right)^{-1} \mathcal{A}\right) .
$$

The same calculations for Subcase 2 give

$$
\sum_{\alpha \leqslant v_{\kappa}<\beta} I_{\alpha, \beta}^{[\kappa]}=\frac{\pi}{n} \psi_{b}(b-) \cdot \operatorname{tr}\left(\mathcal{Q}^{[\kappa]}\left(\widehat{W}^{[\kappa]}\right)^{-1} \mathscr{B}\right) .
$$

Since (27) gives

$$
\mathcal{S}(q)=\frac{1}{2 \pi} \sum_{\alpha \neq \beta} I_{\alpha, \beta}=\frac{1}{2 \pi} \sum_{\kappa=1}^{2}\left(\sum_{\alpha>v_{\kappa} \geqslant \beta} I_{\alpha, \beta}^{[\kappa]}+\sum_{\alpha \leqslant v_{\kappa}<\beta} I_{\alpha, \beta}^{[\kappa]}\right),
$$

formula (6) follows immediately from (35) and (36).

Equation (7) will be proved in the next subsection.

3.2. Linear algebra calculations. In this subsection we skip the index $\kappa$ for the sake of brevity.

3.2.1. Proof of relation (7). We begin with expanding $\mathcal{P}$ and $\mathcal{Q}$ into series. Consider two rows

$$
v_{k}=\left(1, \rho^{k}, \rho^{2 k}, \ldots, \rho^{(v-1) k}, 0, \ldots, 0\right)
$$


and

$$
u_{k}=\left(0, \ldots, 0, \rho^{v k}, \rho^{(v+1) k}, \ldots, \rho^{(n-1) k}\right) .
$$

Denote $\mathcal{P}_{(k)}=\bar{u}_{k}^{T} v_{k}$ and $\mathcal{Q}_{(k)}=\bar{v}_{k}^{T} u_{k}$. Then it is easy to verify that

$$
\mathcal{P}=-\lim _{r \rightarrow 1-} \sum_{k=0}^{\infty} r^{k} \mathcal{P}_{(k)} \quad \text { and } \quad \mathcal{Q}=-\lim _{r \rightarrow 1-} \sum_{k=0}^{\infty} r^{k} \mathcal{Q}_{(k)},
$$

and therefore

$$
\operatorname{tr}\left(\mathcal{P} \widehat{\mathcal{W}}^{-1} \mathcal{A}\right)=-\lim _{r \rightarrow 1-} \sum_{k=0}^{\infty} r^{k} \operatorname{tr}\left(\mathcal{P}_{(k)} \widehat{\mathcal{W}}^{-1} \mathcal{A}\right)
$$

and

$$
\operatorname{tr}\left(\mathcal{Q} \widehat{W}^{-1} \mathscr{B}\right)=-\lim _{r \rightarrow 1-} \sum_{k=0}^{\infty} r^{k} \operatorname{tr}\left(\mathcal{Q}_{(k)} \widehat{w}^{-1} \mathscr{B}\right)
$$

For any $k \in \mathbb{Z}$ and $j \in\{0,1, \ldots, n-1\}$ the direct calculation gives

$$
\left(\mathcal{A} \bar{u}_{k}^{T}\right)_{j+1}=a_{j}\left(\rho^{\nu\left(d_{j}-k\right)}+\rho^{(v+1)\left(d_{j}-k\right)}+\cdots+\rho^{(n-1)\left(d_{j}-k\right)}\right)
$$

and

$$
\left(\widehat{\mathcal{W}} \bar{v}_{k}^{T}\right)_{j+1}=a_{j}\left(1+\rho^{d_{j}-k}+\rho^{2\left(d_{j}-k\right)}+\cdots+\rho^{(\nu-1)\left(d_{j}-k\right)}\right) .
$$

This implies

$$
\mathcal{A} \bar{u}_{k}^{T}+\widehat{w} \bar{v}_{k}^{T}=\sum_{j=0}^{n-1} \sigma\left(k, d_{j}\right) n a_{j} e_{j+1},
$$

where $e_{j}$ is $j$-th vector of standard basis, while

$$
\sigma(x, y)= \begin{cases}1, & x \equiv y \quad(\bmod n) \\ 0, & \text { otherwise }\end{cases}
$$

From (38) we conclude that

$$
\begin{aligned}
\operatorname{tr}\left(\mathcal{P}_{(k)} \widehat{\mathfrak{W}}^{-1} \mathcal{A}\right) & =\operatorname{tr}\left(v_{k} \widehat{\mathbb{W}}^{-1} \mathcal{A} \bar{u}_{k}^{T}\right) \\
& =v_{k} \widehat{\mathbb{W}}^{-1} \mathcal{A} \bar{u}_{k}^{T} \\
& =-v_{k} \bar{v}_{k}^{T}+n \sum_{j=0}^{n-1} \sigma\left(k, d_{j}\right) a_{j} v_{k} \widehat{\mathfrak{W}}^{-1} e_{j+1} \\
& =-v+n \sum_{j=0}^{n-1} \sigma\left(k, d_{j}\right) a_{j} v_{k} \widehat{\mathfrak{W}}^{-1} e_{j+1} .
\end{aligned}
$$


The same calculations give

$$
\operatorname{tr}\left(\mathcal{Q}_{(k)} \widehat{\mathcal{W}}^{-1} \mathcal{B}\right)=-(n-v)+n \sum_{j=0}^{n-1} \sigma\left(k, d_{j}\right) b_{j} u_{k} \widehat{\mathbb{W}}^{-1} e_{j+1}
$$

Since $\sigma\left(k, d_{j}\right)\left(a_{j} v_{k}+b_{j} u_{k}\right)=\sigma\left(k, d_{j}\right) e_{j+1}^{T} \mathcal{W}, j \in\{0,1, \ldots, n-1\}$, formulas (37), (39), and (40) imply

$$
\begin{aligned}
\operatorname{tr} & \left(\mathcal{P} \widehat{\mathcal{W}}^{-1} \mathcal{A}\right)+\operatorname{tr}\left(\mathcal{Q} \widehat{\mathcal{W}}^{-1} \mathcal{B}\right) \\
& =-\lim _{r \rightarrow 1-} \sum_{k=0}^{\infty} r^{k}\left(\operatorname{tr}\left(\mathcal{P}_{(k)} \widehat{\mathcal{W}}^{-1} \mathcal{A}\right)+\operatorname{tr}\left(\mathcal{Q}_{(k)} \widehat{\mathcal{W}}^{-1} \mathcal{B}\right)\right) \\
& =\lim _{r \rightarrow 1-} \sum_{k=0}^{\infty} r^{k}\left(n-n \sum_{j=0}^{n-1} \sigma\left(k, d_{j}\right)\right) \\
& =\lim _{r \rightarrow 1-}\left(\frac{n}{1-r}-n \sum_{j=0}^{n-1} \frac{r^{d_{j}}}{1-r^{n}}\right) \\
& =\lim _{r \rightarrow 1-}\left(\frac{n}{1-r}-\frac{n^{2}}{1-r^{n}}+n \sum_{j=0}^{n-1} \frac{1-r^{d_{j}}}{1-r^{n}}\right) \\
& =\sum_{j=0}^{n-1} d_{j}-\frac{n(n-1)}{2},
\end{aligned}
$$

and (7) follows.

3.2.2. Proof of relation (8). Now we consider the case of almost separated boundary conditions. First, let $n=2 m$.

We introduce three sets:

$$
\begin{aligned}
I & =\left\{k \geqslant 0: k \equiv d_{j} \quad(\bmod n) \text { for some } j<m\right\}, \\
I_{1} & =\left\{d_{0}, d_{1}, \ldots, d_{m-1}\right\}, \\
I_{2} & =\{0, \ldots, 2 m-1\} \backslash I_{1} .
\end{aligned}
$$

For all $k \geqslant 0$ the rows $v_{k}$ lie in the subspace $\operatorname{Span}\left\{e_{j+1}^{T} \mathcal{W}: j \in\{0,1, \ldots, m-1\}\right\}$. Therefore, $v_{k} \widehat{\mathfrak{W}}^{-1} e_{j+1}=0$ for $j \geqslant m$. 
If $k \in I$, then $k \equiv d_{j}(\bmod n)$ for a unique $j<m$. Hence $a_{j} v_{k}=e_{j+1}^{T} \widehat{W}$ and

$$
\sum_{j=0}^{m-1} \sigma\left(k, d_{j}\right) a_{j} v_{k} \widehat{w}^{-1} e_{j+1}^{T}=1 .
$$

Thus, by (39), $\operatorname{tr}\left(\mathcal{P}_{(k)} \widehat{W}^{-1} \mathcal{A}\right)=m$ for $k \in I$.

On the other hand, $\operatorname{tr}\left(\mathcal{P}_{(k)} \widehat{W}^{-1} \mathcal{A}\right)=-m$ for $k \notin I$, as $\sigma\left(k, d_{j}\right)=0$ for all $j<m$.

By (37), we obtain

$$
\begin{aligned}
\operatorname{tr}\left(\mathcal{P} \widehat{W}^{-1} \mathcal{A}\right) & =-\lim _{r \rightarrow 1-}\left(\sum_{k \in I} r^{k} m-\sum_{0 \leqslant k \notin I} r^{k} m\right) \\
& =-m \lim _{r \rightarrow 1-}\left(\sum_{k \in I} r^{k}-\sum_{k \notin I} r^{k}\right) \\
& =-m \lim _{r \rightarrow 1-}\left(\sum_{k \in I_{1}} \frac{r^{k}}{1-r^{2 m}}-\sum_{k \in I_{2}} \frac{r^{k}}{1-r^{2 m}}\right) \\
& =m \lim _{r \rightarrow 1-}\left(\sum_{k \in I_{1}} \frac{1-r^{k}}{1-r^{2 m}}-\sum_{k \in I_{2}} \frac{1-r^{k}}{1-r^{2 m}}\right) \\
& =\frac{1}{2}\left(\sum_{k \in I_{1}} k-\sum_{k \in I_{2}} k\right) \\
& =\sum_{j=0}^{m-1} d_{j}-\frac{m(2 m-1)}{2} .
\end{aligned}
$$

Applying the same calculations to the second term in (6), we prove (8).

3.2.3. Proof of relation (9). Now let $n=2 m+1$. For $\kappa=2$ the previous arguments run almost without changing and give

$$
\operatorname{tr}\left(\mathcal{P}^{[2]}\left(\hat{\mathfrak{W}}^{[2]}\right)^{-1} \mathcal{A}\right)=\sum_{j=0}^{m-1} d_{j}-m^{2} .
$$

The same calculations give

$$
\operatorname{tr}\left(\mathcal{Q}^{[1]}\left(\widehat{\mathcal{W}}^{[1]}\right)^{-1} \mathscr{B}\right)=\sum_{j=m+1}^{2 m-1} d_{j}-m^{2} .
$$

Substituting these formulas into (6) and taking into account (7) we arrive at (9). 
3.2.4. Proof of relation (10). Without loss of generality, we can assume that

$$
a_{j}=1, \quad b_{j}=\vartheta, \quad d_{j}=j, \quad j \in\{0, \ldots, n-1\} .
$$

One can easily check that

$$
\widehat{W}^{-1} e_{j+1}^{T}=\frac{1}{n}\left(1, \rho^{-j}, \ldots, \rho^{-(v-1) j}, \frac{1}{\vartheta} \rho^{-v j}, \ldots, \frac{1}{\vartheta} \rho^{-(n-1) j}\right)^{T},
$$

so $\sigma(k, j) n v_{k} \widehat{W}^{-1} e_{j+1}^{T}=\sigma(k, j) \nu$. By (39), for every $k \geqslant 0$ we have

$$
\operatorname{tr}\left(\mathcal{P}_{(k)} \widehat{W}^{-1} \mathcal{A}\right)=0
$$

Thus we obtain $\operatorname{tr}\left(\mathcal{P} \widehat{W}^{-1} \mathcal{A}\right)=0$. Similarly, $\operatorname{tr}\left(\mathcal{Q} \widehat{W}^{-1} \mathscr{B}\right)=0$, and (10) follows.

\section{Appendix}

We need two technical statements. The first one is a variant of the Riemann-Lebesgue lemma.

Proposition 1. Suppose $q \in L^{1}[a, b], \Gamma \subset\{z \in \mathbb{C}:|z|=1\}$. Let $k_{1}, k_{2} \in \mathbb{C}$ satisfy $k_{1} \neq 0$ and $\operatorname{Re}\left(i w\left(k_{1} x+k_{2}\right)\right) \leqslant 0$ for all $x \in[a, b]$ and $w \in \Gamma$. Then the following relation holds uniformly for $w \in \Gamma$.

$$
\int_{a}^{b} q(x) e^{i R w\left(k_{1} x+k_{2}\right)} d x \rightarrow 0, \quad R \rightarrow+\infty .
$$

Proof. Fix some $\varepsilon>0$. Let a function $q_{1} \in C^{1}([a, b])$ satisfy $q_{1}(a)=q_{1}(b)=0$ and

$$
\int_{a}^{b}\left|q-q_{1}\right| \leqslant \frac{\varepsilon}{2}
$$

Then for $R$ large enough the following estimate holds:

$$
\begin{aligned}
\left|\int_{a}^{b} q_{1}(x) e^{i R w\left(k_{1} x+k_{2}\right)} d x\right| & =\left|\frac{1}{i R w k_{1}} \int_{a}^{b} q_{1}^{\prime}(x) e^{i R w\left(k_{1} x+k_{2}\right)} d x\right| \\
& \leqslant \frac{1}{R\left|k_{1}\right|} \int_{a}^{b}\left|q_{1}^{\prime}\right| \\
& <\frac{\varepsilon}{2} .
\end{aligned}
$$


Trivial estimate

$$
\left|\int_{a}^{b}\left(q(x)-q_{1}(x)\right) e^{i R w\left(k_{1} x+k_{2}\right)} d x\right| \leqslant \int_{a}^{b}\left|q-q_{1}\right| \leqslant \frac{\varepsilon}{2}
$$

completes the proof.

The second statement concerns the function $f(R w)$ introduced by formula (15).

Proposition 2. There exists some constant $M>0$ such that for all $R>0$

$$
\int_{\Gamma_{1} \cup \Gamma_{2}} R f(R w)|d w|<M .
$$

Proof. We need to estimate several integrals of the same type. Most of them are exponentially small because the real part of the index is strictly less than zero on the whole arc $\Gamma_{1} \cup \Gamma_{2}$. There are few integrals where the real part of the index tends to zero on the end of the arc. We write estimates for one of such integrals:

$$
\begin{aligned}
\int_{\Gamma_{1} \cup \Gamma_{2}} R\left|e^{i R w(b-a)}\right||d w| & =\int_{0}^{\frac{\pi}{n}} R e^{-R(b-a) \sin \varphi} d \varphi \\
& \leqslant \int_{0}^{\frac{\pi}{n}} R e^{-\frac{2}{\pi} R(b-a) \varphi} d \varphi \\
& <\frac{\pi}{2(b-a)} .
\end{aligned}
$$

The other ones are estimated in the same way.

\section{References}

[1] A. Bayramov, Z. Oer, S. Öztürk Uslu, and S. Kizilbudak C̣aliṣkan, On the regularized trace of a fourth order regular differential equation. Int. J. Contemp. Math. Sci. 1 (2006), 245-254. MR 2289032 Zbl 1165.34049

[2] I. M. Gelfand and B. M. Levitan, On a simple identity for the eigenvalues of a second-order differential operator. Doklady Akad. Nauk SSSR (N.S.) 88 (1953), 593-596. In Russian. MR 0056157 Zbl 0053.06001

[3] A. Haar, Zur Theorie der orthogonalen Funktionensysteme. (1) Math. Ann. 69 (1910), 331-371; (2) Math. Ann. 71 (1911), 38-53. MR 1511592 (1) MR 1511640 (2)

JFM 41.0469.03 (1) JFM 42.0433.01 (2) 
[4] C. J. A. Halberg Jr. and V.A. Kramer, A generalization of the trace concept. Duke Math. J. 27 (1960), 607-617. MR 0115091 Zbl 0095.09502

[5] E. W. Hobson, On a general convergence theorem, and the theory of the representation of a function by a series of normal functions. Proc. London Math. Soc. (2) 6 (1908), 349-395. MR 1575133 JFM 39.0476.02

[6] A. I. Kozko and A. S. Pechentsov, Regularized traces of higher-order singular differential operators. Mat. Zametki 83 (2008), 39-49. English transl., Math. Notes 83 (2008), 37-47. MR 2399996 Zbl 1159.34354

[7] A. I. Kozko and A. S. Pechentsov, Regularized traces of singular differential operators of order $2 m$. Sovr. Probl. Mat. Mech. 3 (2009), 45-57. In Russian.

[8] A. G. Kostyuchenko, On some spectral properties of differential operators. Sci.D. dissertation. Lomonosov Moscow State University, Moscow, 1966. In Russian.

[9] A. G. Kostyuchenko, Asymptotic behavior of the spectral function of a singular differential operator of order $2 m$. Dokl. Akad. Nauk SSSR 168 (1966) 276-279. English transl., Soviet Math. Dokl. 7 (1966), 632-635. MR 0202008 Zbl 0156.31202

[10] B. M. Levitan and I .S. Sargsyan, Introduction to spectral theory: selfadjoint ordinary differential operators. Nauka, Moscow, 1970. English transl. by A. Feinstein, Translations of Mathematical Monographs 39. American Mathematical Society, Providence, R.I., 1975. MR MR0299863 (Russian) MR 0369797 (transl.) Zbl 0225.47019 (Russian) Zbl 0302.47036 (transl.)

[11] V. B. Lidskii, Non-self-adjoint operators with a trace. Dokl. Akad. Nauk SSSR 125 (1959) 485-487. English transl., Am. Math. Soc., Transl., II. Ser. 47 (1965), 43-46. MR 0105023 Zbl 0158.14702

[12] A. Minkin, Equiconvergence theorems for differential operators. J. Math. Sci. (New York) 96 (1999), 3631-3715. MR 1724439 Zbl 0951.47046

[13] M. A. Naimark, Linear differential operators. Second ed. revised and augmented. With an appendix by V. È. Ljance. Nauka, Moscow, 1969. English transl. of the first ed., Linear differential operators. Part I. Elementary theory of linear differential operators. Frederick Ungar Publishing, New York, N.Y., 1967. MR 0353061 (Russian) MR 216050 (transl.) Zbl 0219.34001 (transl.)

[14] A. I. Nazarov, D. M. Stolyarov, and P. B. Zatitskiy, Following traces of V. A. Sadovnichii. Preprints of St.-Petersburg Math. Society 4, 2010. In Russian.

[15] A. I. Nazarov, D. M. Stolyarov, and P. B. Zatitskiy, Formula of regularized traces. Dokl. Akad. Nauk 442 (2012), 162-165. English transl., Dokl. Math. 85 (2012), 29-32. MR 2962174 Zbl 1242.47036

[16] V. A. Sadovnichii, The trace of ordinary differential operators of high order. Mat. Sb. (N.S.) 72 (1967), 293-317. English transl., Math. USSR-Sb. 1 (1967), 263-288. MR 0209553 Zbl 0166.34504

[17] V. A. Sadovnichii, A. S. Pechentsov, and A. I. Kozko, Regularized traces of singular differential operators. Dokl. Akad. Nauk 427 (2009), 461-465. English transl., Dokl. Math. 80 (2009), 550-554. MR 2584580 Zbl 1190.47053

[18] V. A. Sadovnichii and V. E. Podolskii, Traces of operators. Uspekhi Mat. Nauk 61 (2006), 89-156. English transl., Russian Math. Surveys 61 (2006), 885-953. MR 2328258 Zbl 1157.47013 
[19] A. M. Savchuk ond A. A. Shkalikov, Trace formula for the Sturm-Liouville operators with singular potentials. Mat. Zametki 69 (2001), 427-442. English transl., Math. Notes 69 (2001), 387-400. MR 1846840 Zbl 1005.34077

[20] R. F. Shevchenko, On the trace of a differential operator. Dokl. Akad. Nauk SSSR 164 (1965), 62-65. English transl., Soviet Math. Dokl. 6 (1965), 1183-1186. MR 0186858 Zbl 0144.10202

[21] A. A. Shkalikov, Boundary-value problems for ordinary differential equations with a parameter in the boundary conditions. Trudy Sem. Petrovsk. 9 (1983), 190-229. English transl., J. Soviet Math. 33 (1986), 1311-1342. MR 731903 Zbl 0553.34014

[22] V. A. Stekloff, Sur les expressions asymptotiques de certains fonctions définies par des équations différéntielles linéaieres de deuxiéme ordre, et leurs applications au problème du developpement d'une fonction arbitraires en séries procédant suivantes dites fonctions. Kharkov. Soobtscheniya matem. obtschesstva. 10 (1907), 97-199. JFM 38.0310 .02

[23] V. A. Stekloff, Solution générale du problème de developpement d'une fonction arbitraire en séries suivant les fonctions fondamentales de Sturm-Liouville. Rend. Acad. Lincei 19 (1910), 490-496. JFM 41.0360.03

[24] J. D. Tamarkin, On some general problems of the theory of ordinary linear differential operators and on expansion of arbitrary function into series. Petrograd, 1917. In Russian.

Received December 29, 2012; revised June 17, 2013

Alexander I. Nazarov, St. Petersburg Department of Steklov Institute, Fontanka, 27, St. Petersburg, 191023, Russia

St. Petersburg State University, Universitetskii pr. 28, St. Petersburg, 198504, Russia

E-mail: al.il.nazarov@gmail.com

Dmitry M. Stolyarov, Chebyshev Laboratory, St. Petersburg State University, $14^{\text {th }}$ Line V.O., 29b, Saint Petersburg, 199178, Russia

St. Petersburg Department of Steklov Institute, Fontanka, 27, St. Petersburg, 191023, Russia

E-mail: dms@pdmi.ras.ru

Pavel B. Zatitskiy, Chebyshev Laboratory, St. Petersburg State University, $14^{\text {th }}$ Line V.O., 29b, Saint Petersburg, 199178, Russia

St. Petersburg Department of Steklov Institute, Fontanka, 27, St. Petersburg, 191023, Russia

E-mail: paxa239@yandex.ru 